\title{
Quasi-exact solution of the Riemann problem for generalised dam-break over a mobile initially flat bed
}

\author{
4 Fangfang Zhu • Nicholas Dodd
}

6 Received: date / Accepted: date

\begin{abstract}
This paper investigates dam-break problems with flows on one or two sides of zero or nonzero velocities over a mobile initially flat bed, and quasi-exact solutions are presented by solving the Riemann problems using the simple wave theory. The flow structures after dam collapse for nonzero velocities are much richer than those for zero velocities on both sides, although they are also a combination of waves of different characteristic families, which are consistent with [7]. The wave can be a rarefaction, a shock, or a combination of a rarefaction and a semi-characteristic shock. The semi-characteristic shock is related to the morphodynamic characteristics. The relationship between morphodynamic and hydrodynamic characteristics is illustrated, along with types of wave (shock, rarefaction or a combination of these), and sediment convergence and type of characteristic. It is shown that the types of waves that may occur in the Riemann solution, and, in some cases, their possible approximate location, can be determined prior to the construction of the Riemann solution itself. The Riemann solution presented here can be used to study shock-shock interactions.
\end{abstract}

Keywords Dam-break · Mobile bed · Simple wave $\cdot$ Shallow water equations · Quasi-exact solution

Natural Science Foundation of China (Grant No. 51509135)

Fangfang Zhu

Department of Civil Engineering

University of Nottingham Ningbo China

Ningbo, 315100, China

E-mail: Fangfang.Zhu@nottingham.edu.cn

Nicholas Dodd

Faculty of Engineering

University of Nottingham

Nottingham NG7 2RD, UK 


\section{Introduction}

Dam failure can cause catastrophic flooding, and urban areas or farmlands downstream can be dramatically affected. In addition, a dam-break flow can cause huge erosion and deposition. Forecasting of the floods due to dam-breaks is necessary for an emergency evacuation from the flooded area to prevent loss of life and huge damages. In addition to its practical significance, the dambreak problem provides the simplest available model for a number of important phenomena, e.g., river flows and swash flows [11,10,23,21]. Thus, dam-break phenomena have been one main research interest for many years [14].

Nonlinear shallow water equations (NSWEs), which have often been used for describing one or two dimensional dam-break flows [15, 13, 16,1,4,18] (see [9] for a discussion of the validity of these equations.). The exact and quasiexact solutions for dam-break problems can provide us with information about common shallow water flows, which can also be used as verification cases for numerical solvers [17]. Dam-break problems can be classified into those with water on both left and right sides (wet-wet problems) and those with water only on the left side (wet-dry problems), over initially continuous or discontinuous beds. The exact solutions for 1D dam-break problems over a flat fixed continuous bed with various velocities on both sides are well known $[13,15$, 16]. The $1 \mathrm{D}$ wet-wet dam-break problem on a fixed flat bed with a discontinuous bottom geometry, was further examined by [1], and exact solutions were presented.

Here we extend this class of solutions so as to consider non-zero initial velocities on a mobile bed. These problems are of practical as well as theoretical interest, because they are closely related to shock-shock interactions which commonly occur in river flows and shallow water flows on a beach [8]. In shock-shock interactions, when two stable shocks coalesce, they form a new discontinuity [19]. The new discontinuity is usually not stable, and would collapse. This discontinuity corresponds to a dam-break problem of non-zero initial velocities. [22] applied the simple wave theory [3] to a restricted class of Riemann problems: wet-dry and also wet-wet dam-break problems over beds of initially continuous or discontinuous bed levels. However, they only considered zero initial velocities on both sides. In reality, commonly occurring flows on beaches deviate from this when a following, larger wave encounters the temporarily halted earlier wave, either as a wet-wet or wet-dry problem. In these circumstances, typically, $\hat{u}_{l}>0 \geq \hat{u}_{r}$, where $\hat{u}_{l}$ and $\hat{u}_{r}$ are water velocities on the left and right side of the dam; see Fig. 1. Additionally, and even more generally, when one larger shock overtakes a smaller one, a Riemann problem is also generated, because the new configuration is unstable: see Fig. 1.

Therefore, here we consider this more general case, in which we allow for $\hat{u}_{l} \neq \hat{u}_{r} \neq 0$. We also assume $\hat{h}_{l} \gg \hat{h}_{r} \geq 0$, where $\hat{h}_{l}$ and $\hat{h}_{r}$ are water depths on the left and right side of the dam, consistent with these flows and dambreak flows in general. Finally, for simplicity, we restrict ourselves to cases in which $\hat{B}_{l}=\hat{B}_{r}=0$, where $\hat{B}_{l}$ and $\hat{B}_{r}$ are bed levels on the left and right side of the dam. The bed therefore has no initial slope or discontinuity. 
(a)

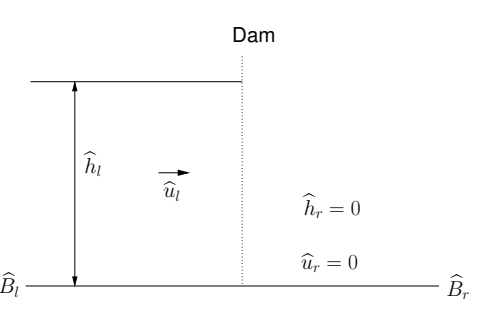

(b)

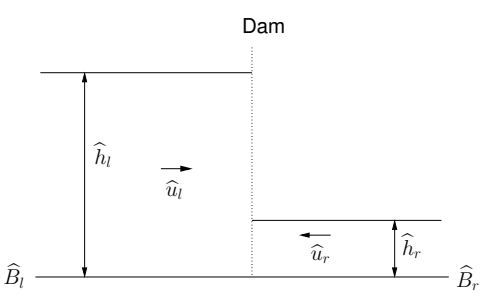

Fig. 1 Schematic diagram of the initial configuration for dam-break problems. (a): Wet-dry dam-break problem; (b) wet-wet dam-break problem. $\hat{h}$ represents water depth $(\mathrm{m}), \hat{u}$ is a depth-averaged horizontal velocity $\left(\mathrm{ms}^{-1}\right)$ and $\hat{B}$ is bed level $(\mathrm{m})$. The subscripts $l$ and $r$ indicate the left and right side of the dam.

In the next section we present the model equations. We then present the quasi-exact dam-break solutions in Sect. 3, and finally, we present our conclusions in Sect. 4.

\section{Model development}

\subsection{Governing equations}

The nonlinear shallow water equations and the Exner equation including only bed load are utilised to describe the dam-break flow

$$
\begin{aligned}
\hat{h}_{\hat{t}}+\hat{u} \hat{h}_{\hat{x}}+\hat{h} \hat{u}_{\hat{x}} & =0, \\
\hat{u}_{\hat{t}}+\hat{u} \hat{u}_{\hat{x}}+g \hat{h}_{\hat{x}}+g \hat{B}_{\hat{x}} & =0, \\
\hat{B}_{\hat{t}}+\xi \hat{q}_{\hat{x}} & =0,
\end{aligned}
$$

where $\hat{x}$ represents horizontal distance $(\mathrm{m}), \hat{t}$ is time $(\mathrm{s}), \hat{h}$ represents water depth $(\mathrm{m}), \hat{u}$ is a depth-averaged horizontal velocity $\left(\mathrm{ms}^{-1}\right), \hat{B}$ is bed level $(\mathrm{m}), \hat{q}$ is sediment flux due to bed load $\left(\mathrm{m}^{2} \mathrm{~s}^{-1}\right)$ and $g$ is acceleration due to gravity $\left(\mathrm{ms}^{-2}\right) \cdot \xi=\frac{1}{1-p}$ with $p$ being the porosity. In Fig. 1 we illustrate the situation being considered.

We use the Grass formula $\hat{q}=\hat{A} \hat{u}^{3}[2]$ to describe the sediment transport rate as bed load [5,23], with $A$ being the bed mobility parameter $\left(\mathrm{s}^{2} \mathrm{~m}^{-1}\right)$.

Therefore, (3) becomes

$$
\hat{B}_{\hat{t}}+3 \xi \hat{A} \hat{u}^{2} \hat{u}_{\hat{x}}=0 .
$$

\subsection{Non-dimensionalization}

The nondimensional variables are

$$
x=\frac{\hat{x}}{\hat{h}_{0}}, t=\frac{\hat{t}}{\hat{h}_{0}^{1 / 2} g^{-1 / 2}}, h=\frac{\hat{h}}{\hat{h}_{0}}, u=\frac{\hat{u}}{\hat{u}_{0}}, \text { and } B=\frac{\hat{B}}{\hat{h}_{0}},
$$


where $\hat{h}_{0}$ is a length scale, which is usually taken to be the higher of the two initial depths, and $\hat{u}_{0}=\left(g \hat{h}_{0}\right)^{1 / 2}$.

Substituting (5) into the governing equations (1), (2) and (4) gives

$$
\begin{aligned}
h_{t}+u h_{x}+h u_{x} & =0, \\
u_{t}+u u_{x}+h_{x}+B_{x} & =0, \\
B_{t}+3 \sigma u^{2} u_{x} & =0
\end{aligned}
$$

90 where $\sigma=\xi \hat{A} g$ is a non-dimensional parameter related to bed mobility.

The vector form of these three non-dimensional governing equations is

$$
\vec{U}_{t}+\mathbf{A}(\vec{U}) \vec{U}_{x}=0
$$

$91 \quad$ with

$$
\vec{U}=\left[\begin{array}{l}
h \\
u \\
B
\end{array}\right], \mathbf{A}(\vec{U})=\left[\begin{array}{ccc}
u & h & 0 \\
1 & u & 1 \\
0 & 3 \sigma u^{2} & 0
\end{array}\right]
$$

The eigenvalues of $\mathbf{A}$ are the roots of the polynomial equation

$$
\lambda^{3}-2 u \lambda^{2}+\left(u^{2}-3 \sigma u^{2}-h\right) \lambda+3 \sigma u^{3}=0 .
$$

The polynomial equation (11) has three roots, which are denoted $\lambda_{1}, \lambda_{2}$ and $\lambda_{3}$ such that $\lambda_{1} \leq \lambda_{3} \leq \lambda_{2}$. For the solution of $\lambda_{1}, \lambda_{2}$ and $\lambda_{3}$ we refer to [4,5]. For nonzero depth, when $u>0$, we have $\lambda_{1}<0<\lambda_{3}<u<\lambda_{2}$; while when $u=0$, we have $\lambda_{1}<0=\lambda_{3}=u<\lambda_{2}$; when $u<0$, we have $\lambda_{1}<u<\lambda_{3}<0<\lambda_{2}$.

Let $\lambda^{\prime}=\lambda / \sqrt{h}$; Eq. (11) can then be rearranged into a characteristic polynomial for $\lambda^{\prime}$, which depends only on Froude number $F=u / \sqrt{h}$ and $\sigma$ :

$$
{\lambda^{\prime}}^{3}-2 F{\lambda^{\prime 2}}^{2}+\left((1-3 \sigma) F^{2}-1\right) \lambda^{\prime}+3 \sigma F^{3}=0 .
$$

We plot $\lambda^{\prime}$ versus $F$ for $\sigma=0.01$ in Fig. 2, which will be used in Sect. 3 to help explain the structure of the Riemann solutions. In the morphodynamic system defined by Eq. (12) we define a characteristic, $\lambda^{\prime}$, as being hydrodynamic if $\lambda^{\prime} \approx \lambda^{\prime}{ }_{+,-}$, where $\lambda^{\prime}{ }_{+,-}$are the characteristics in the equivalent hydrodynamic system (Eq. (12) with $\sigma=0$ ). Accordingly, if a characteristic $\lambda^{\prime} \not \lambda^{\prime}{ }_{+,-}$, then it is defined as a morphodynamic characteristic $\left(\lambda^{\prime}{ }_{m}\right)$, which is assumed to be related to a bed wave. Note that $\lambda^{\prime}{ }_{m} \approx 0$ because the bed change at the hydrodynamic time scale is negligible [22]; see Fig. 2. Also note that $\lambda^{\prime} \approx \lambda^{\prime}{ }_{+,-} \equiv \lambda^{\prime} \approx F \pm 1 \Leftrightarrow \lambda \approx u \pm h^{1 / 2}$. The relationship between $\frac{d \lambda^{\prime}}{d F}$ and $\frac{d \lambda}{d F}$ is derived in Sect. 2.5. 


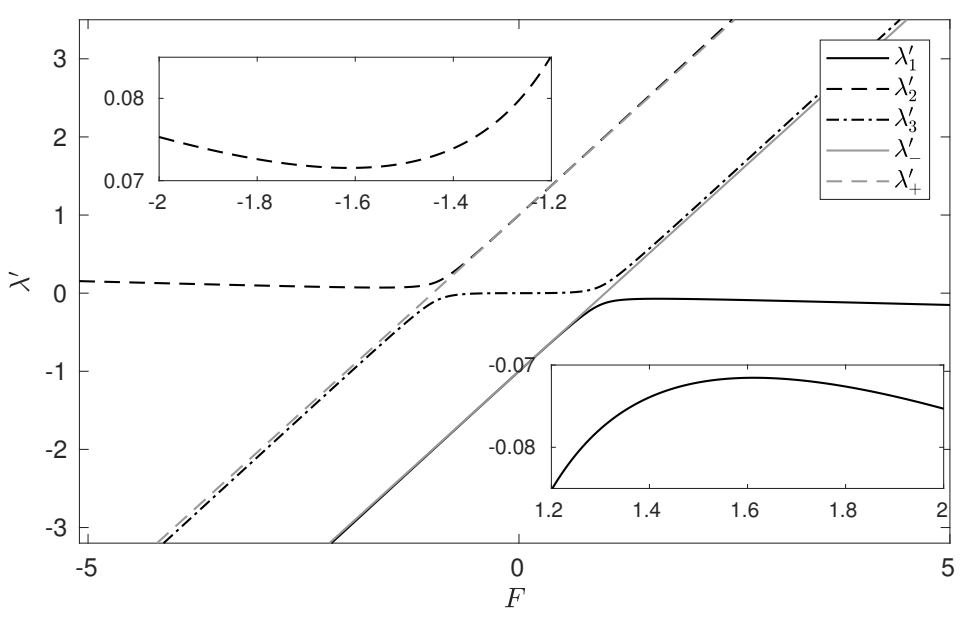

Fig. 2 Dimensionless characteristic velocities for our system with $\sigma=0.01$ (after [21], figure 2). $\lambda_{+,-}^{\prime}=\lambda_{+,-} / \sqrt{h}$ with $\lambda_{+,-}$being the equivalent hydrodynamic (fixed bed) characteristic velocities. The insets, which show a close-up of $\lambda^{\prime}-F$ space, illustrate the non-monotonic behaviour of the $\lambda_{1}$ and $\lambda_{2}$ characteristics.

\subsection{Initial conditions}

The initial conditions for a general dam-break problem are shown in Fig. 1.

As mentioned in Sect. 1 we consider general $u_{l}$ and $u_{r}$. We also assume $h_{l} \gg h_{r}$, and thus consider only initial depths consistent with classical dambreak flows. Accordingly, we set $h_{l}=1$ and $h_{r}=0.1$ for all the examined wet-wet dam-break problems. The wet-dry dam-break problem is the limiting case of wet-wet dam-break problem, and for this we take $h_{r}=0$. Finally, we set $B_{l}=B_{r}=0$. The bed is erodible with $\sigma=0.01$, consistent with [22].

\subsection{Methodology}

As the dam-break problem investigated in this paper is essentially a Riemann problem, it can be solved using simple wave theory [3,7]. Across a simple wave, i.e., a rarefaction fan, we have $[3,22]$ :

$$
\begin{aligned}
d u & =\frac{\lambda_{i}-u}{h} d h, \\
d B & =\left(\frac{\left(\lambda_{i}-u\right)^{2}}{h}-1\right) d h .
\end{aligned}
$$

We refer the readers to [22] for the application of simple wave theory in solving dam-break problems. 
We use the following shock conditions as necessary

$$
\begin{aligned}
h_{R} u_{R}-h_{L} u_{L}-\left(h_{R}-h_{L}\right) W & =0, \\
W\left(h_{R} u_{R}-h_{L} u_{L}\right)-\left(h_{R} u_{R}^{2}+\frac{h_{R}^{2}}{2}-h_{L} u_{L}^{2}-\frac{h_{L}^{2}}{2}\right)-\int_{B_{L}}^{B_{R}} h d B & =0, \\
\left(B_{R}-B_{L}\right) W-\sigma\left(u_{R}^{3}-u_{L}^{3}\right) & =0,
\end{aligned}
$$

where $L$ and $R$ represent variables on the left and right side of a shock, and $W$ is shock velocity.

We take the approximation proposed by [8] for the term $\int_{B_{L}}^{B_{R}} h d B$ in (16):

$$
\int_{B_{L}}^{B_{R}} h d B \approx \frac{1}{2}\left(B_{R}-B_{L}\right)\left(h_{R}+h_{L}\right) .
$$

Note that for morphodynamic shocks we could use that of [22]. That approximation is necessary for the large initial bed changes considered therein, but not for this case.

\subsubsection{Wave structure determination}

For a general Riemann problem of $n$ equations, there are $n$ waves associated with the $n$ characteristic families [7]. Therefore, for the wet-wet dam-break problems there are 3 waves separated by 2 newly formed constant regions. We refer to these regions as left and right "star" regions, and variables in them as $\mathbf{U}_{l *}$ and $\mathbf{U}_{r *}$, to distinguish them from the constant initial regions $\left(\mathbf{U}_{l}\right.$ and $\left.\mathbf{U}_{r}\right)$.

However, it should be noted that for wet-dry dam-break problems over a mobile bed, there are only 2 waves separated by 1 newly formed constant ("star") region, the variables in which are denoted $\mathbf{U}_{*}$. One wave vanishes because of the presence of the dry bed [22].

The task is to find $\mathbf{U}_{*}$, or $\mathbf{U}_{l *}$ and $\mathbf{U}_{r *}$, and identify the wave types. The waves could be rarefactions, or shocks or semi-characteristic shocks [22]. The characteristic configuration of each wave type is shown in Fig. 3. For the wetwet problem, firstly we give initial estimates for $h_{l *}$ and $h_{r *}$, and then we assume the wave structures according to the estimates. Secondly, we verify our assumption by obtaining the Riemann solution. For example, we can first assume a wave is a rarefaction fan, and if the Riemann solution shows the divergence of characteristics across this wave, then this assumption is true. If characteristics converge, then it must be a shock instead of a rarefaction. If the characteristics diverge across some part of the wave and converge in some other part, then a multi-valued problem occurs, and a rarefaction fan together with a semi-characteristic shock is introduced. Finally, we refine $h_{l *}$ and $h_{r *}$ by checking the Riemann solution. 
(a)

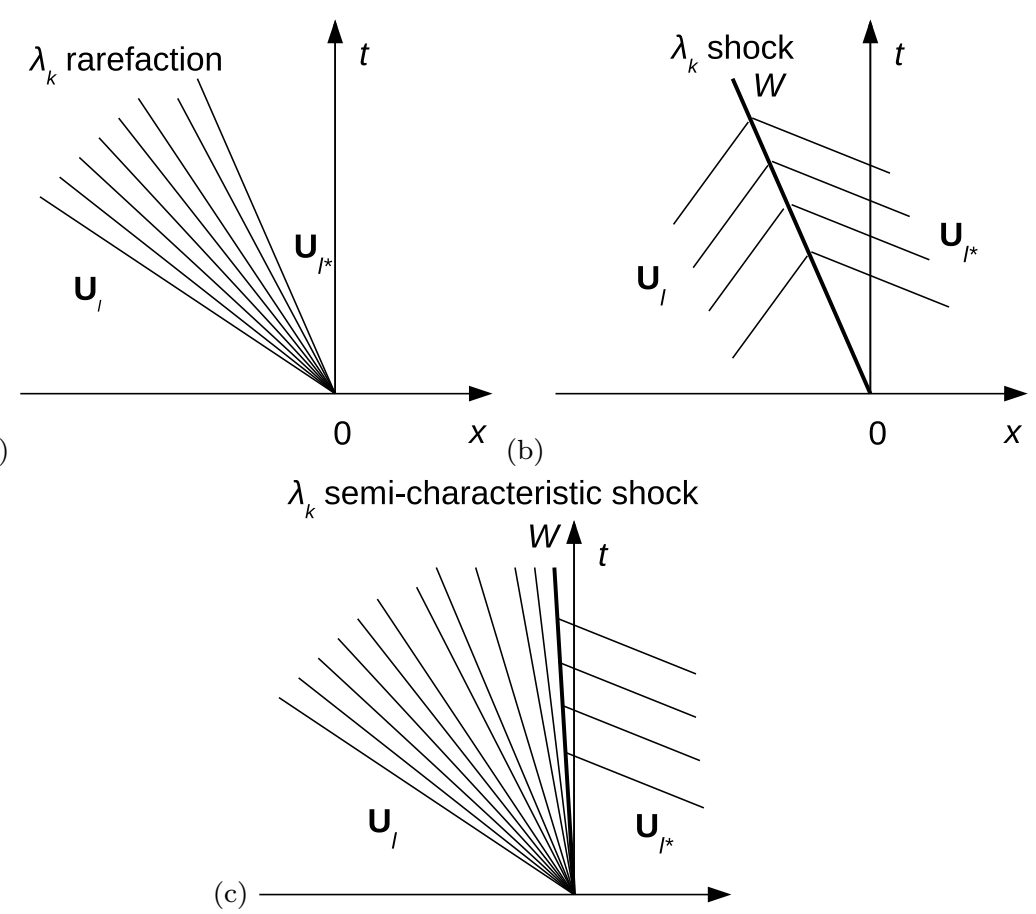

Fig. 3 Schematic diagrams depicting characteristic configurations for (a) rarefaction, (b) shock and (c) semi-characteristic shock.

\subsubsection{Computation procedure}

The computation procedures for a wet-wet dam-break problem are as follows:

(i) Estimate initial values for $h_{l *}$ and $h_{r *}$.

(ii) Assume wave types for the $\lambda_{1,2,3}$ waves according to $h_{l *}$ and $h_{r *}$. They could be rarefactions, shocks or combinations of a rarefaction and a semicharacteristic shock of the same family.

(iii) Find wave solutions. Using the assumed $h_{l *}$ and $h_{r *}$, and assumed wave structures, we construct the Riemann solution for some finite time, $t>0$, using (13) and (14) for rarefactions and (15)-(17) for shocks to obtain a structure for the $\lambda_{1,2,3}$ waves.

(iv) Refine $h_{l *}$ and $h_{r *}$

- Compare the $u$ values calculated or already known in one designated constant region. This region could be the right (left) constant region if the Riemann problem is solved from left (right) to right (left), or the left or right star regions if solved from both the left and right. If the two $u$ values do not agree to the desired level of accuracy, $h_{r *}\left(h_{l *}\right)$ is changed, i.e. $h_{r *}^{(1)}\left(h_{l *}^{(1)}\right)$. Then the wave solutions are recalculated and $u$ values again found (Step ii-iii). This process is repeated until the desired accuracy is achieved via the bisection method; once values 
agree, the correct water depth $h_{r *}\left(h_{l *}\right)$ for the fixed $h_{l *}\left(h_{r *}\right)$ is considered to have been achieved.

- We then check whether the $B$ values calculated or already known in the designated constant region agree to the required accuracy. If this is achieved, the updated values for $h_{l *}$ and $h_{r *}$ are assumed to be correct, and we have arrived at a solution to the Riemann problem. If not, we change the value of $h_{l *}\left(h_{r *}\right)$ and repeat the above steps to the required accuracy.

For wet-dry dam-break problems, the procedures are similar except that there is only one newly formed constant region, and the shock condition for sediment conservation at the tip is used to refine $h_{*}$.

\subsubsection{Wave type determination}

It is shown in Fig. 2 that the characteristics $\lambda^{\prime}{ }_{+,-}$in the hydrodynamic problem increase monotonically as $F$ increases. However, this is not so for $\lambda^{\prime}{ }_{1,2}$. We can see from Fig. 2 that $\lambda_{1,2,3}^{\prime}$ change identity between morphodynamic and hydrodynamic characteristics. $\lambda^{\prime}{ }_{1} \approx \lambda^{\prime}{ }_{-}$when $F<1$, and $\lambda^{\prime}{ }_{3} \approx \lambda^{\prime}{ }_{-}$when $F>1 . \lambda^{\prime}{ }_{3} \approx \lambda^{\prime}+$ when $F<-1$, and $\lambda_{2}^{\prime} \approx \lambda^{\prime}+$ when $F>-1$.

Here, we follow [22] in identifying a morphodynamic (hydrodynamic) wave as being associated with a morphodynamic (hydrodynamic) characteristic. A hydrodynamic shock is thus defined as that caused by the convergence of hydrodynamic characteristics; or the convergence of hydrodynamic and morphodynamic characteristics, but dominated by hydrodynamic characteristics, in the sense that the shock possesses the properties of a hydrodynamic shock (see below). A morphodynamic shock is then defined as that caused by the convergence of morphodynamic characteristics; or a convergence of morphodynamic and hydrodynamic characteristics, but not dominated by hydrodynamic characteristics (i.e., it does not possess the properties of a hydrodynamic shock). We define hydro- and morphodynamic rarefactions in a similar way.

The properties of a $\lambda_{+}\left(\lambda_{-}\right)$wave are that $\lambda_{+}>u\left(\lambda_{-}<u\right)$ so that water flows right to left (left to right) across a $\lambda_{+}\left(\lambda_{-}\right)$wave, relative to the wave. Furthermore, if, as the water flows across the $\lambda_{+}\left(\lambda_{-}\right)$wave, it flows from a region of smaller to larger depth then the water velocity increases (decreases), and the $\lambda_{+}\left(\lambda_{-}\right)$wave is a shock. Conversely, if water velocity decreases (increases) and depth decreases the $\lambda_{+}\left(\lambda_{-}\right)$wave is a rarefaction.

We assume that the hydrodynamic waves in the morphodynamic system behave similarly to those in the hydrodynamic system (i.e., $\sigma=0$ ). Therefore, the properties above are assumed to be valid in the morphodynamic system. For the morphodynamic characteristics, $\lambda_{m}$, we have $0>\lambda_{m}>u$, when $u<0$, and $0<\lambda_{m}<u$, when $u>0$. However, the above analysis is not used for a morphodynamic wave, because the flow across the morphodynamic wave is more complex. $\lambda_{m}^{\prime}$ does not vary monotonically (see Fig. 2) and this gives rise to semi-characteristic shocks, as characteristics first diverge and then converge. We can use the $\lambda^{\prime}-F$ plot to help deduce the wave type. 
across the $i$ th rarefaction fan. Therefore,

$$
\begin{aligned}
\frac{d \lambda^{\prime}}{d F} & =\frac{d \lambda}{d F} h^{-1 / 2}-\frac{1}{2} h^{-3 / 2} \frac{h^{3 / 2}}{\lambda_{i}-\frac{3}{2} u} \lambda \\
\Rightarrow h^{-1 / 2} \frac{d \lambda}{d F} & =\frac{d \lambda^{\prime}}{d F}+\frac{1}{2} \frac{\lambda^{\prime}}{\lambda^{\prime}{ }_{i}-\frac{3}{2} F} .
\end{aligned}
$$

2.5 Determination of semi-characteristic shock position in $\lambda^{\prime}-F$ space

$$
\frac{d \lambda^{\prime}}{d F}=\frac{d \lambda}{d F} h^{-1 / 2}-\frac{1}{2} h^{-3 / 2} \frac{d h}{d F} \lambda
$$

Since $F=F(h, u)$

$$
\begin{aligned}
\frac{d F}{d h} & =\frac{\partial F}{\partial h}+\frac{\partial F}{\partial u} \frac{d u}{d h} \\
& =\frac{\lambda_{i}-\frac{3}{2} u}{h^{3 / 2}},
\end{aligned}
$$

Note that here $\lambda$ and $\lambda^{\prime}$ denote any characteristic, but $\lambda^{\prime}{ }_{i}$ refers specifically to the $i$ th rarefaction fan.

Now, from simple wave theory [3] we know that across the $i$ th rarefaction fan $\frac{d \lambda_{i}}{d h}=\frac{d \lambda_{i}}{d F} \frac{d F}{d h}$. Therefore,

$$
h^{1 / 2} \frac{d \lambda_{i}}{d h}=\left(\frac{d \lambda_{i}^{\prime}}{d F}+\frac{1}{2} \frac{\lambda^{\prime}{ }_{i}}{\lambda_{i}^{\prime}-\frac{3}{2} F}\right)\left(\lambda_{i}^{\prime}-\frac{3}{2} F\right) .
$$

We know that if $\frac{d \lambda_{i}}{d h}=0$ then a semi-characteristic shock can potentially form. Therefore, because in general $h>0$ we can, using (22), place these locations in $\left(\lambda^{\prime}, F\right)$ space, which correspond to locations where

$$
\frac{d \lambda^{\prime}}{d F}=-\frac{1}{2} \frac{\lambda_{i}^{\prime}}{\lambda_{i}^{\prime}-\frac{3}{2} F} \text { or } \lambda_{i}^{\prime}=\frac{3}{2} F
$$

Note that if $\lambda_{i}^{\prime}=\frac{3}{2} F$, then we must also have $\lambda_{i}=0$. This, in theory, could occur for $\lambda_{i}=\lambda_{3}$. From [22] we also know that, without loss of generality,

$$
\frac{d \lambda_{i}}{d h}=\nabla_{\vec{U}} \lambda_{i} \cdot \vec{R}
$$

where $\vec{R}$ are right eigenvectors of $\mathbf{A}$ in Eq. (10). Furthermore, developing from $[22]^{1}$ we have

$$
h^{1 / 2} \frac{d \lambda_{i}}{d h}=\frac{\lambda_{i}^{\prime}+\left(2{\lambda^{\prime}}_{i}^{2}-(2-6 \sigma) F{\lambda^{\prime}}_{i}-9 \sigma F^{2}\right)\left(\lambda^{\prime}{ }_{i}-F\right)}{3{\lambda^{\prime}}_{i}^{2}-4 F \lambda_{i}^{\prime}+(1-3 \sigma) F^{2}-1} .
$$

${ }^{1}$ We note that there is a misprint in equation (2.25) of [22]. The factor $\left(u-\lambda_{i}\right)$ should be $\left(\lambda_{i}-u\right)$. 
Hence, equating the right of (25) to zero should also give the (same) positions at which a semi-characteristics shock could occur in a Riemann problem.

Fig. 2 shows that $\frac{d \lambda_{1}^{\prime}}{d F}=0$ occurs at $F \approx 1.613, \frac{d \lambda_{2}^{\prime}}{d F}=0$ occurs at $F \approx-1.613$ and $\frac{d \lambda^{\prime}}{d F}=0$ occurs at $F=0$. We find from Eq. (21) that when $\frac{d \lambda_{1,2}^{\prime}}{d F}=0, \frac{d \lambda_{1,2}}{d F} \neq 0$ because $\lambda_{1,2}^{\prime} \neq 0$. However, $\frac{d \lambda_{3}^{\prime}}{d F}(F=0)=0 \Rightarrow \frac{d \lambda^{\prime}}{d F}=0$ because $\frac{\lambda^{\prime}{ }_{3}}{F} \rightarrow 0$ when $F \rightarrow 0$.

In Fig. 4 we see $h^{1 / 2} \frac{d \lambda_{i}}{d h}$ calculated from both (22) and (25) with $\sigma=$ 0.01 . It can be seen that there are three possible vicinities in which a semicharacteristic shock could occur, and the positions are consistent with those from Fig. 2.

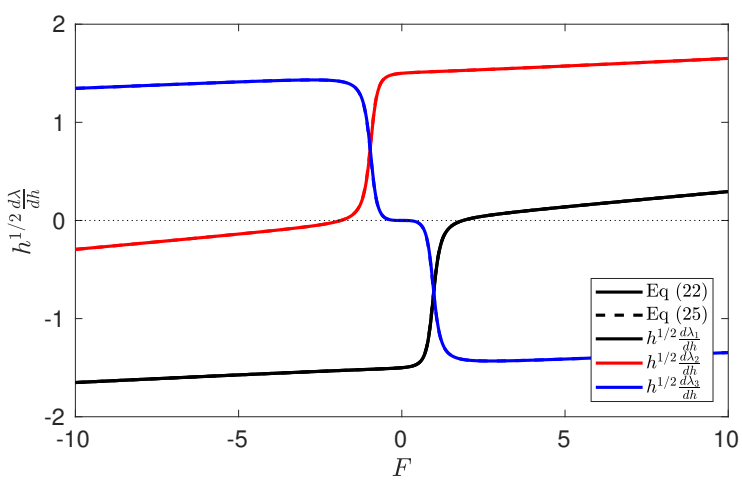

Fig. $4 h^{1 / 2} \frac{d \lambda}{d h}$ calculated from both (22) and (25) with $\sigma=0.01$.

\section{Riemann solutions for dam-break problems}

\subsection{Wet-dry dam-break problem}

The structures of wet-dry dam-break problems over an initially flat erodible bed for general $u_{l}$ are shown in Fig. 5 . There are five types of structure:

(i) $\lambda_{1}$ rarefaction fan, star region of 0 velocity next to $x=0$, and bed step at $x=0$ (e.g. $\left.u_{l}=-2\right)$

(ii) $\lambda_{1}$ rarefaction fan, star region and $\lambda_{3}$ rarefaction (e.g. $\left.u_{l}=-1.5,0,1.5\right)$;

(iii) $\lambda_{1}$ rarefaction fan, $\lambda_{1}$ semi-characteristic shock, star region, and $\lambda_{3}$ rarefaction fan (e.g. $u_{l}=1.83$ );

(iv) $\lambda_{1}$ shock, star region, and $\lambda_{3}$ rarefaction fan (e.g. $u_{l}=2.5$ );

(v) $\lambda_{1}$ rarefaction ( $h$ increases as $x$ increases, in which $u>0$ ), star region, and $\lambda_{3}$ rarefaction (e.g. $\left.u_{l}=3.5\right)$.

When $u_{l}=0$, the wave structure is a $\lambda_{1}$ rarefaction and a $\lambda_{3}$ rarefaction (structure (ii)), which is consistent with that presented by [4]. The $\lambda_{1}$ wave is a combination of a $\lambda_{-}$hydrodynamic wave and a morphodynamic wave; $\lambda_{3}$ 

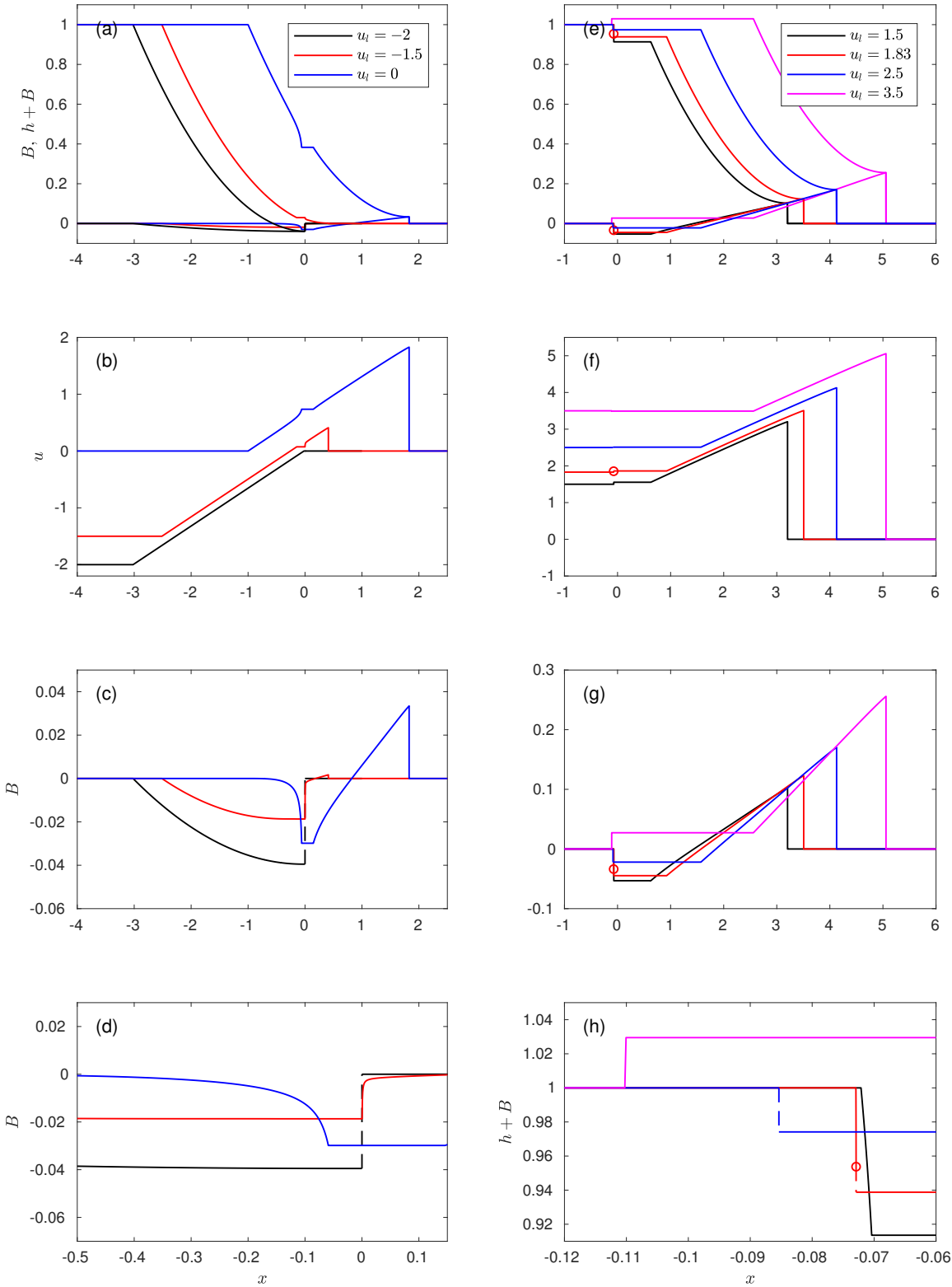

Fig. 5 Structure of the wave solution at $t=1$ for a wet-dry Riemann problem with general $u_{l}$. Dashed lines indicate jumps at shocks or semi-characteristic shocks. $\circ$ separates the rarefaction and semi-characteristic shock of the same wave. (a) and (e) show water surface levels $h+B$ and bed levels $B$, (b) and (f) show water velocities $u$, (c) and (g) show bed levels $B$, and (d) and (h) show magnified bed levels $B$. (a)-(d) correspond to the same dam-break problems, and (e)-(h) correspond to the same dam-break problems.

is a $\lambda_{-}$hydrodynamic wave. The sediment in the $\lambda_{1}$ wave and star region is 
eroded by the right moving water, and is deposited in the $\lambda_{3}$ wave region. The major difference between the structures of (i) and (ii) is whether there is a $\lambda_{3}$ wave. The relative position of the free surface level in the star region $\left(h_{*}+B_{*}\right)$ and the bed level on the right side of the dam $\left(B_{r}\right)$ determines whether there is a $\lambda_{3}$ wave.

From Fig. 5(a), we can see that when $u_{l}=0, h_{*}+B_{*}>B_{r}$. When $u_{l}$ decreases, the water depth $\left(h_{*}\right)$, velocity $\left(u_{*}\right)$ and bed level $\left(B_{*}\right)$ in the star region decrease. For some $u_{l}, h_{*}+B_{*}=B_{r}$. At this point, we must have $u_{*}=0$ and a bed step (discontinuity) forms at $x=0$ (structure (i)) because sediment is moved by the initially left moving water. This is because if $u_{*}>0$, water in the star region would flow towards the bed step on its right and be reflected back resulting in a larger $h_{*}$ such that $h_{*}+B_{*}>B_{r}$. Conversely, if $u_{*}<0$ water moves away from $x=0$ position resulting in a smaller $h_{*}$ and $h_{*}+B_{*}<B_{r}$

When $u_{l}$ further decreases, we have $h_{*}+B_{*}<B_{r}$. The key point is whether a further decrease in $u_{l}$ would result in $u_{*}$ remaining 0 or also decreasing. However, when $u_{*}<0$ and $h_{*}+B_{*}<B_{r}$, the structure is not stable, and $h_{*}$ would decrease such that $u_{*} \rightarrow 0$. Therefore, there is always a star region with $u_{*}=0$ adjacent to $x=0$, implying that water does not leave the discontinuity at $x=0$. It might appear counterintuitive that we should have $u_{*}=0$ for $u_{l} \ll 0$. However, it can be explained by the simple wave theory. For the $\lambda_{1}$ rarefaction wave, we have $d u=\frac{\lambda_{1}-u}{h} d h$, so as $h_{*} \rightarrow 0, \int d u$ is unbounded. Therefore, as $u_{l} \rightarrow-\infty$, we can have $u_{*}=0$. Alternatively, we can note that $\lambda_{1}\left(u_{l}\right)<u_{l}<u_{*}=0$ for all $u_{l}$. This implies that all fluid in the left constant region will eventually enter the $\lambda_{1}$ rarefaction fan, accelerate, and come to rest.

When $u_{l}$ gradually increases from $0, h_{*}$ and $u_{*}$ increase (structure (ii)). We can see from Fig. 5 that the $\lambda_{1}$ rarefaction is more confined when $u_{l}$ increases, which is because the hydrodynamic part gradually disappears. As $u_{l}$ increases further, the $\lambda_{1}$ characteristics in the $\lambda_{1}$ fan first diverge and then converge, and therefore a semi-characteristic shock is introduced for $u_{l}=1.83$ (structure (iii)). The semi-characteristic shock is a morphodynamic wave, and together with the $\lambda_{1}$ fan, it connects the hydrodynamic and morphodynamic characteristics.

When $u_{l}$ further increases, the water depth on the left side of the semicharacteristic shock gradually increases to $h_{l}$ and the $\lambda_{1}$ rarefaction fan disappears. The $\lambda_{1}$ wave is a semi-characteristic shock only for a particular $u_{l}$. In other words, one side of this shock coincides with a $\lambda_{1}$ characteristic, but this characteristic is that of the left constant state. When $u_{l}$ further increases, the $\lambda_{1}$ semi-characteristic shock becomes a $\lambda_{1}$ shock, i.e., structure (iv).

If $u_{l}$ increases even further, $h_{*}$ increases. When $h_{*}=h_{l}$, the $\lambda_{1}$ shock disappears and if $u_{l}$ further increases, $h_{*}>h_{l}$, and the $\lambda_{1}$ wave becomes a rarefaction, across which the water depth increases from left to right (structure $(\mathrm{v})$ ). It should be noted that the $\lambda_{1}$ rarefaction fan in structure (v) is somewhat different from that in structure (ii). In structure (ii), the $\lambda_{1}$ wave is 
a combination of a $\lambda_{-}$hydrodynamic wave and a morphodynamic wave, and in structure (v) it is a morphodynamic wave.

When the $\lambda_{1}$ wave is a morphodynamic wave or consists of a morphodynamic wave, it has a richer pattern. It can be a rarefaction, or a semicharacteristic shock, or a shock, or combinations of these wave types.

The $\lambda_{3}$ wave is always a rarefaction because the $\lambda_{3}$ wave near the tip is always a hydrodynamic wave $\left(\lambda_{-}\right)$and water depth decreases across the $\lambda_{3}$ wave. This is consistent with finding of [4] that a dry bed cannot be adjacent to a shock. However, it should be noted, also consistent with [4], that there is a sediment bore at the tip, with water depth of zero on both sides, and only $u$ and $B$ vary across it.

See Sect. A for the interpretation of this solution in $\left(\lambda^{\prime}, F\right)$ space.

\subsection{Wet-wet dam-break problem}

First we include a small depth of water $\left(h_{r}=0.1\right)$ on the previously dry region, to examine the difference this makes to the Riemann solution. Then we take $u_{l}=0$ and vary $u_{r}$ to illustrate how varying velocity on the low side affects the wave structure.

\subsection{1 $u_{r}=0$}

The wave structures of a wet-wet dam-break problem over a flat erodible bed with $u_{r}=0$ but various $u_{l}$ are shown in Fig. 6 . As might be expected, analogies with the wet-dry case are apparent. The six types of structure are:

(i) $\lambda_{1}$ rarefaction, left star region, $\lambda_{3}$ shock, right star region, $\lambda_{2}$ semicharacteristic shock and $\lambda_{2}$ rarefaction (e.g., $u_{l}=-2$ );

(ii) $\lambda_{1}$ rarefaction, left star region, $\lambda_{3}$ shock, right star region, and $\lambda_{2}$ rarefaction (e.g., $u_{l}=-1.65$ );

(iii) $\lambda_{1}$ rarefaction, left star region, $\lambda_{3}$ shock, right star region, and $\lambda_{2}$ shock (e.g., $\left.u_{l}=-1.33\right)$

(iv) $\lambda_{1}$ rarefaction, left star region, $\lambda_{3}$ rarefaction, right star region, and $\lambda_{2}$ shock (e.g., $u_{l}=-1$ and 0 );

(v) $\lambda_{1}$ rarefaction ( $h$ increases as $x$ increases, in which $u>0$ ), left star region, $\lambda_{3}$ rarefaction, right star region, and $\lambda_{2}$ shock (e.g., $u_{l}=2$ ).

(vi) $\lambda_{1}$ rarefaction ( $h$ increases as $x$ increases, in which $u>0$ ), left star region, $\lambda_{3}$ shock, right star region, and $\lambda_{2}$ shock (e.g., $u_{l}=2.5$ ).

The solutions in Fig. 6 mostly have clear analogues in Fig. 5. Structure (i) can be seen to be equivalent to (i) of the wet-dry case, in which ponded water occurs. That structure exists for $u_{l} \lesssim-1.695$ in the wet-dry case, whereas the present structure does so for $u_{l} \underset{\lesssim}{\lesssim}-1.692$. The still water on the right now drains into the eroded (right star) region via a $\lambda_{2}$ rarefaction and semicharacteristic shock. This $\lambda_{2}$ wave is a combination of a hydrodynamic and morphodynamic characteristic, and it is on the morphodynamic portion that 

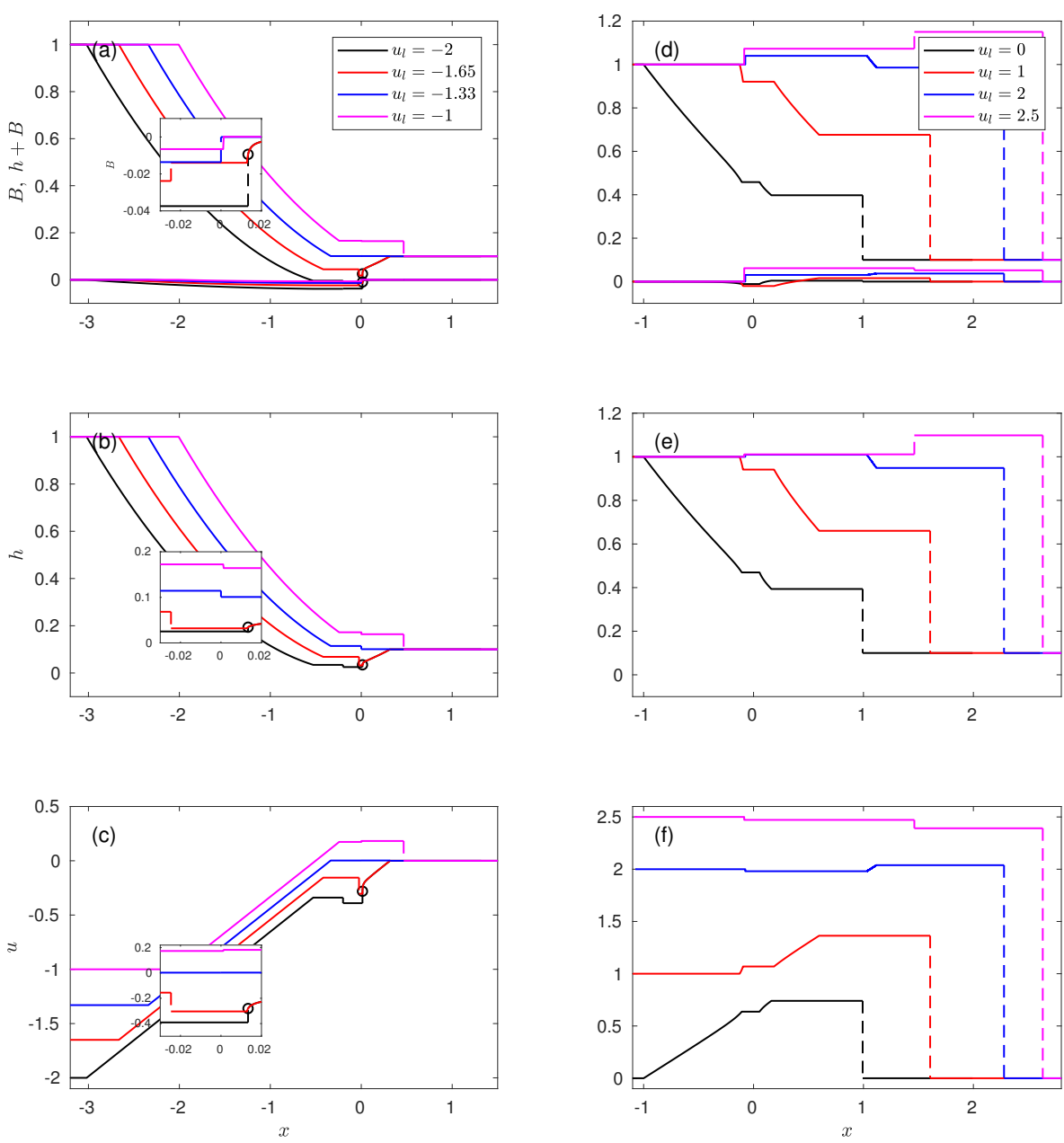

Fig. 6 Structure of the wave solution at $t=1$ for a wet-wet Riemann problem with general $u_{l}$ and $u_{r}=0$. Dashed lines indicate jumps at shocks or semi-characteristic shocks. $\circ$ separates the rarefaction and semi-characteristic shock of the same wave. (a) and (d) show water surface levels $h+B$ and bed levels $B$, (b) and (e) show water depths $h$, and (c) and (f) show water velocities $u$. (a)-(c) correspond to the same dam-break problems, and (d)-(f) correspond to the same dam-break problems.

the convergence of characteristics occurs (see Sect. B). The rapid bed change occurs on the morphodynamic portion, as flow moves from being sub- to supercritical.

For an increased but still negative $u_{l}$, structure (ii) emerges. Here, the $\lambda_{2}$ wave terminates before a characteristic convergence can occur, and hence it is a rarefaction only. Erosion is reduced, and now occurs across both $\lambda_{2}$ wave and $\lambda_{3}$ shock, the latter being partly morphodynamic.

When $u_{l} \approx-1.334, u_{l *}=u_{r *}=0, h_{l *}>h_{r *}=h_{r}$ and $h_{l *}+B_{l *}=h_{r *}+$ $B_{r *}$. The $\lambda_{2}$ wave becomes confined to one point because $h_{r *}=h_{r}$, and the $\lambda_{3}$ 
wave is a stationary shock because $u_{l *}=u_{r *}=0$ and $h_{l *}+B_{l *}=h_{r *}+B_{r *}$. So, $u_{l} \approx-1.334$ is the value above which flow to the right is possible. Structure (iii) occurs as the hydrostatic pressure drives flow from left to right. So, initially left moving water in the left constant region, enters the $\lambda_{1}$ rarefaction, accelerates across that wave such that it acquires a positive velocity, and then enters the $\lambda_{3}$ wave before accelerating across that into the right star region, where it remains as the $\lambda_{2}$ shock proceeds to the right. The $\lambda_{3}$ wave is a shock with $W>0$ when $-1.334 \lesssim u_{l} \lesssim-1.33$ (structure (iii)). When $u_{l} \gtrsim-1.33$, the $\lambda_{3}$ shock becomes a rarefaction, i.e., structure (iv). Structure (iv) is familiar to us because it is the structure for dam-break problem of $u_{l}=u_{r}=0$.

When $u_{l}$ increases from 0 to a positive value, water on the left side moves immediately towards the right, causing water to accumulate, and $h_{l *}$ and $h_{r *}$ both to increase with $h_{r *} \rightarrow h_{l *} \rightarrow 1$. At the same time, $u_{l *}$ and $u_{r *}$ also increase. The hydrodynamic portion in the $\lambda_{1}$ wave gradually decreases, and the morphodynamic portion increases. Finally, the $\lambda_{1}$ wave becomes a morphodynamic wave. When $h_{l *}>1, \lambda_{1}$ wave is still a rarefaction, but $h$ increases as $x$ increases. This is similar to the equivalent wet-dry problem solution in Fig. 5. The wave structure becomes structure (v). When $u_{l}$ further increases, $1<h_{l *}<h_{r *}$, and the $\lambda_{3}$ wave becomes a shock, in which $W>0$ (structure (vi)). This $\lambda_{3}$ shock is a hydrodynamic $\left(\lambda_{-}\right)$shock with an increase in water depth from left to right. Thus, the water decelerates across a morphodynamic $\lambda_{1}$ wave, and again does so across a right-moving $\lambda_{3}$ hydrodynamic shock, before entering (and remaining in) the right star region, where it is joined by water from the right constant state as the $\lambda_{2}$ hydrodynamic shock moves rapidly to the right.

\subsection{2 $u_{l}=0$}

The wave structures of dam-break with $u_{l}=0$ and varying $u_{r}$ are shown in Fig. 7. There are four types of structure:

(i) $\lambda_{1}$ shock, left star region, $\lambda_{3}$ shock, right star region, $\lambda_{2}$ rarefaction (e.g., $\left.u_{r}=-2.5,-1.934\right)$

(ii) $\lambda_{1}$ shock, left star region, $\lambda_{3}$ shock, right star region, and $\lambda_{2}$ shock (e.g. $\left.u_{r}=-1.933\right)$

(iii) $\lambda_{1}$ rarefaction, left star region, $\lambda_{3}$ rarefaction, right star region, and $\lambda_{2}$ shock (e.g., $\left.u_{r}=-1,0\right)$;

(iv) $\lambda_{1}$ rarefaction, left star region, $\lambda_{3}$ rarefaction, right star region, and $\lambda_{2}$ rarefaction (e.g., $u_{r}=1.5$ ).

For large negative $u_{r}$ (structure (i)) (Fig. 7(a)-(c)) this large speed means that the change in momentum of this flow overcomes the hydrostatic pressure gradient and flow ensues from right to left ( $u \leq 0$ across the Riemann solution). There is therefore a decrease in $|F|$ from right to left. Across the $\lambda_{2}$ wave, again, from right to left, there is a small increase in $h$ and a modest decrease in $|u|$, which results in sediment convergence across the fan and the creation of a substantial bed-step. Note that flow is still supercritical on the step. Transition 

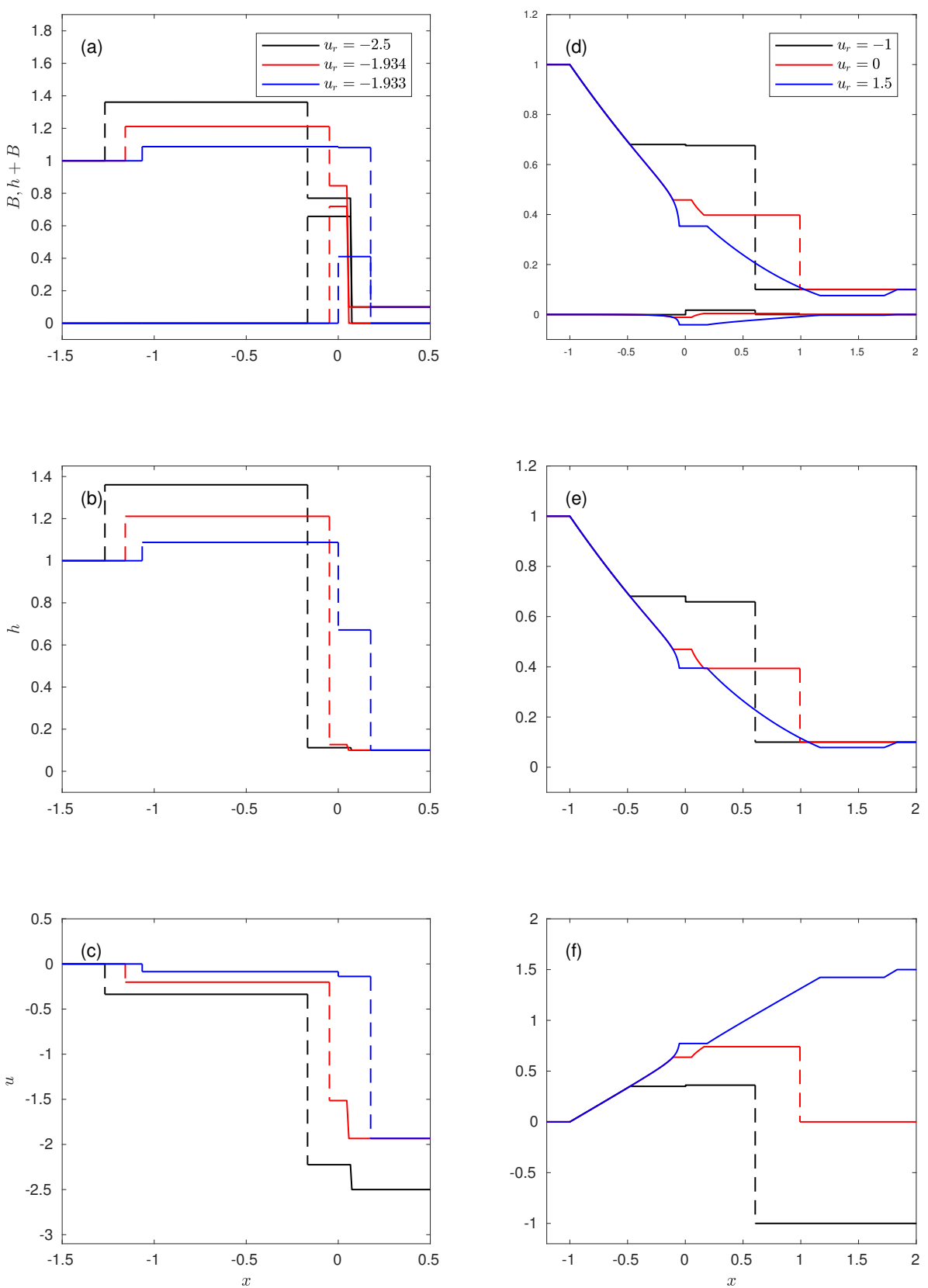

Fig. 7 Structure of the wave solution at $t=1$ for a wet-wet Riemann problem with varying $u_{r}$ and $u_{l}=0$. Dashed lines indicate jumps at shocks or semi-characteristic shocks. (a) and (d) show water surface levels $h+B$ and bed levels $B$, (b) and (e) show water depths $h$, and (c) and (f) show water velocities $u$. (a)-(c) correspond to the same dam-break problems, and (d)-(f) correspond to the same dam-break problems. 
to sub-critical flow occurs across the $\lambda_{3}$ (shock) wave, and so further sediment convergence occurs. Thus, the bed-step advances both up- and downstream as it accumulates sediment. On the left side a shock wave (with negligible bed change) advances into the still water. Note that, as in the wet-dry case, in terms of wave structure, the mapping of this Riemann solution into $\left(\lambda^{\prime}, F\right)$ space (see Sect. C) once more yields the same types of waves as those obtained from the solution itself (see Fig. 10).

As $u_{r}$ increases this structure persists until $u_{r} \approx-1.934$ (Fig. $7(\mathrm{a})-(\mathrm{c})$ ). For a further increase, $u_{r} \approx-1.933$, the structure is transformed to structure (ii) (Fig. 7(a)-(c)). This happens because the $\lambda_{2}$ wave now becomes a shock, with the smaller $\left|u_{r}\right|$ allowing an abrupt flow change across this wave. Now, the change from super- to sub-critical flow occurs across the $\lambda_{2}$ wave.

Note the very large change in $u_{r *}$ as $u_{r}$ varies between these two values, which differ by about $0.05 \%$. The corresponding $\lambda_{2}$ wave changes from a morphodynamic wave into a hydrodynamic wave, and $\lambda_{3}$ shock changes from a hydrodynamic shock into a morphodynamic shock. This accounts for the abrupt change. Further note that the bed-step created by this sediment convergence now advances more rapidly upstream than downstream. For velocity $W$ of the $\lambda_{3}$ shock: $0<|W| \ll 1$. This abrupt change is further investigated numerically in Sect. D.

As $u_{r}$ increases further a $\lambda_{1}$ rarefaction emerges, which yields structure (iii). This apparently minor change (Fig. 7) actually accompanies a flow reversal with $u_{* r}>u_{* l}>0$, as the fluid in the left constant region enters the right star region across the $\lambda_{1,3}$ waves because $\lambda_{1,3}<u$. There is therefore a decrease in $h$ across the Riemann solution, and an increase in $u$ across the $\lambda_{1,3}$ waves as the water is driven across the $\lambda_{1,3}$ waves by the pressure gradient. The $\lambda_{3}$ wave also becomes a rarefaction. The bed on the left side is eroded, and deposited on the right. The right moving water then encounters the relatively slower right moving water in the right constant region. This results in the convergence of $\lambda_{2}$ characteristics, and therefore the $\lambda_{2}$ wave is a shock. Across the $\lambda_{2}$ shock, water jumps from the right to the left side, thus gaining velocity. Therefore, $h$ and $u$ both decrease from left to right side. Sediment convergence mostly takes place at the leading (right) edge as it propagates to the right. Note also that convergence is also much reduced because of the much smaller change in $u$ across the $\lambda_{2}$ wave.

When $u_{r}$ increases from 0 to a positive value, $h_{l *}$ and $h_{r *}$ decrease because water on the right side is moving away from $x=0$; when $h_{r *}<h_{r}$, the $\lambda_{2}$ wave changes from a shock into a rarefaction (structure (iv)) and water on the right constant region is overtaken by the right edge of the $\lambda_{2}$ rarefaction fan and therefore enters the right star region.

\section{Conclusion}

Dam-break problems with flows on one or two sides with zero or nonzero velocities on an initially flat mobile bed have been investigated, and quasi- 
analytical solutions are presented with examples. The solutions are consistent with previous studies $[7,17,4,22]$.

The solutions are consistent with the theory proposed by [7] that for a Riemann problem of $n$ equations there are in general $n$ waves associated with the $n$ characteristic families. The solutions presented are, therefore, of more varied structure than the equivalent hydrodynamic ones. In particular, as noted by [22], solutions sometimes contain a semi-characteristic shock, rather than just shocks and rarefactions.

The characteristics can be classified as hydrodynamic characteristics and morphodynamic characteristics. The transition between diverging hydrodynamic and morphodynamic characteristics is usually through a fan, which consists of a hydrodynamic part and a morphodynamic part, and a semicharacteristic shock often occurs when there is a large change in the characteristics (Fig. 3(c)). The semi-characteristic shock is a morphodynamic wave.

The possible position of these semi-characteristic shocks can be determined without solving the Riemann problem. This immediately indicates the kinds of waves that can occur in the Riemann solution, and is very useful for solving the problem. If this property extends to a broader range of functional relationships of the form $\hat{q}=\hat{q}(\hat{h}, \hat{u})[12,20]$, then it will have even greater utility.

It is also noted that, there may be an abrupt change of wave structure, associated with which there is a transition between morphodynamic wave and hydrodynamic wave.

By far the largest observed changes in bed level in these dam-break problems is for the case of a highly supercritical flow (small depth) flowing into a body of water of much larger depth. If this inflow is large enough it can cause overall flow in the direction of the inflow, and a large deposition is formed across a $\lambda_{2}$ morphodynamic rarefaction (of low speed, opposing the inflow). This deposition region terminates in a $\lambda_{3}$ shock, which advances slowly in the inflow direction. This bedform is reminiscent of the bed-step observed by [6], and simulated by [21]. This depositional feature is then potentially available to be transported / entrained should the inflow subsequently diminish. The Riemann solution in this work can provide theoretical basis for shock-shock interaction in the swash zone.

In real flows, the threshold of motion may have an effect on the wave structure after the dam collapse. However, for the class of flows in the swash region of fine sand, which motivates this work, the effect of a threshold is insignificant [21] (Appendix A). Therefore, the effect of threshold of motion is not investigated in the present work.

Acknowledgements This work is supported by Natural Science Foundation of China with the project code 51509135, and the authors would like to acknowledge for their financial support. The authors would also like to acknowledge University of Nottingham UK and Ningbo China. 


\section{A Interpretation of wave structures in Sect. 3.1 by characteristics}

Because $\lambda^{\prime}=\frac{\lambda}{\sqrt{h}}$ (see Fig. 2) is dependent only on $F$ it is instructive to map each of the profiles in Fig. 5 onto it: see Fig. 8.
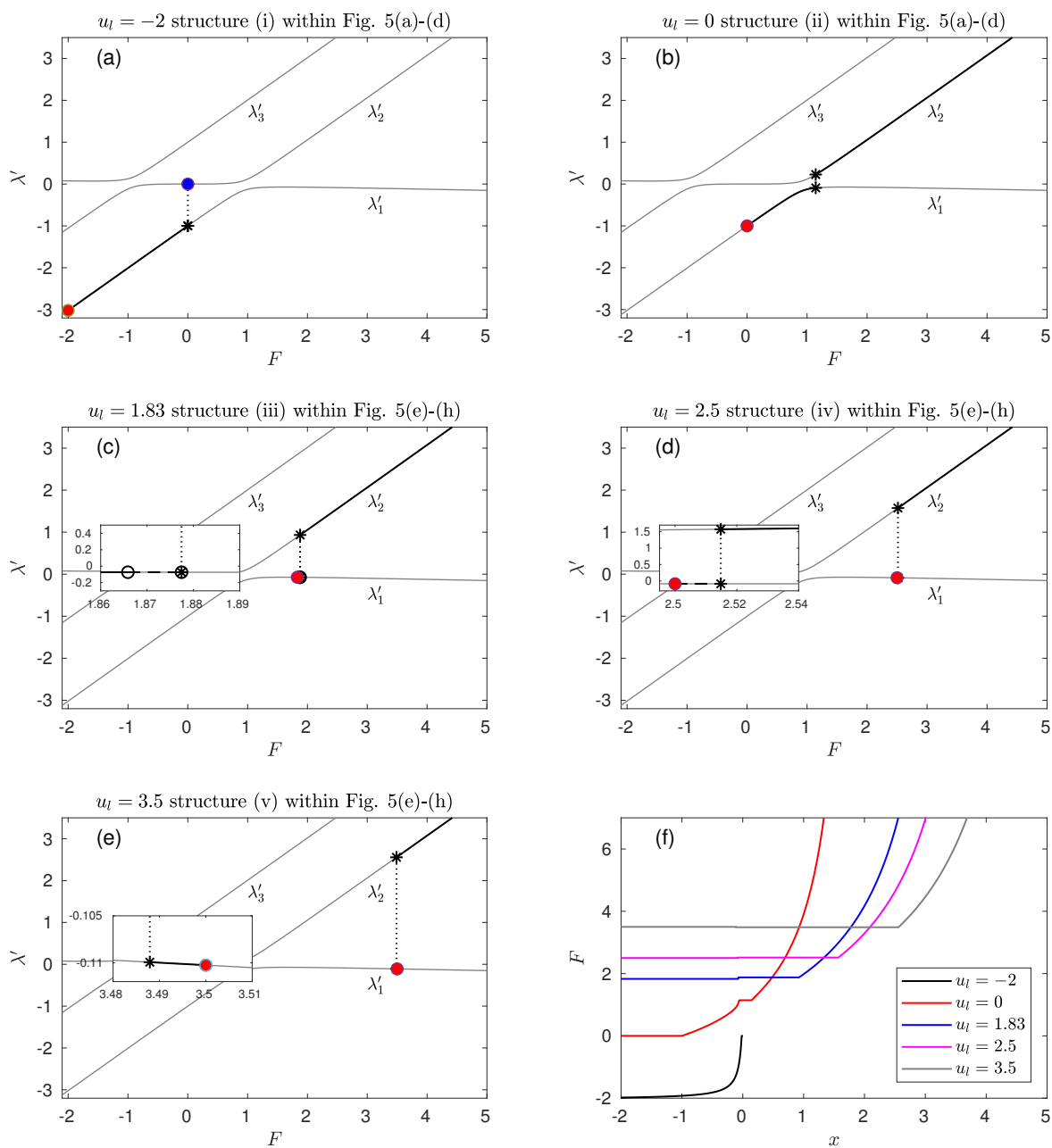

Fig. 8 (a)-(e): Illustrations of the Riemann solutions depicted in Fig. 5 in $\left(\lambda^{\prime}, F\right)$ space as the solutions are traversed from left (indicated by the red filled circle) to right (indicated by the blue filled circle; note, however, that this circle is only visible for $u_{l}=-2$, because for other structures it is located in the limit $F \rightarrow \infty$. Dashed lines indicate jumps at shocks or semi-characteristic shocks. o separates the rarefaction and semi-characteristic shock of the same wave. Dotted lines with black * represent jumps from different characteristic families of waves in star regions. (f): Illustration of how $F$ varies across these solutions.

It can immediately be seen that each solution begins on the left at $F=F_{l}$ on the $\lambda^{\prime}{ }_{1}$ dispersion curve, and each solution will, at some point, jump to the $\lambda^{\prime}{ }_{3}$ curve, and then proceed to the right as $F \rightarrow \infty$, apart from structure (i), which terminates at $F=0$. 
The question is then at what point the jump occurs. In $\lambda^{\prime}-F$ space, this, along with any other jump due to the presence of a shock, completely describes the solution. Note that for structures (i)-(iv), $h(u)$ is monotonic decreasing (increasing) across the Riemann solution from left to right, notwithstanding the jump to zero $u$ on the dry side. Therefore, $F$ is monotonic increasing for positive $u$. $F$ could be increasing or decreasing for negative $u$. However, for all the examined negative $u_{l}$ values, $F$ is monotonic increasing (Fig. 8(f)). Further note that $\frac{d \lambda_{1}^{\prime}}{d F}=0$ at $F \approx 1.613, \Rightarrow$ for $F<1.613, \lambda^{\prime}{ }_{1}$ increases for increasing $F$ (Fig. 2).

For $u_{r}=0$ in structure (ii), the jump occurs for $F<1.613$ (see Fig. 8). In this region $\frac{d \lambda^{\prime}{ }_{1}}{d F}>0, \Rightarrow \frac{d \lambda_{1}}{d F}>0$ too, because $h$, as noted, is monotonic decreasing and $\lambda^{\prime}{ }_{1}<0$. Therefore, the $\lambda_{1}$ wave is a rarefaction. We mentioned in Sect. 3.1 that the $\lambda_{1}$ wave is a combination of a $\lambda_{-}$hydrodynamic wave and a morphodynamic wave, which can also be seen from Fig. 5(b). The characteristics analysis that $\frac{d \lambda_{1}}{d F}>0$ is consistent with the analysis from physical perspective that when water depth decreases across the $\lambda_{-}$wave, it is a rarefaction.

When $F>1.613$, in contrast, we are in the region $\frac{d \lambda^{\prime}}{d F}<0$. Because $\lambda^{\prime}{ }_{1}$ and $h$ are both decreasing it is not obvious whether $\frac{d \lambda_{1}}{d F} \lessgtr 0$ in each case. We notice that when $F>1$, the $\lambda_{1}\left(\lambda^{\prime}{ }_{1}\right)$ characteristics behave as morphodynamic characteristics. It should be noted that $\frac{d \lambda_{1}^{\prime}}{d F}$ is a small value around $F=1.613$, and according to Eq. (21) in Sect. 2.5 it is possible that $\frac{d \lambda^{\prime} 1}{d F}$ and $\frac{d \lambda_{1}}{d F}$ have different signs.

The results show that for $1.613 \lesssim u_{l} \lesssim 1.83, \lambda_{1}$ increases across the $\lambda_{1}$ wave, and therefore the $\lambda_{1}$ wave is a rarefaction fan (structure (ii)). In this case, $\frac{d \lambda^{\prime}}{d F}<0$ and $\frac{d \lambda_{1}}{d F}>0$. However, for a further increase in $u_{l}$ we have $\frac{d \lambda_{1}}{d F}>0$ in some part of the $\lambda_{1}$ wave, and $\frac{d \lambda_{1}}{d F}<0$ in the other part. The results show that when $1.83 \lesssim u_{l} \lesssim 1.848, \lambda_{1}$ characteristics first diverge and then converge. Therefore, the $\lambda_{1}$ wave is a combination of $\lambda_{1}$ rarefaction and a $\lambda_{1}$ semi-characteristic shock (structure (iii), e.g., $u_{l}=1.83$ in Fig. $5(\mathrm{e})-(\mathrm{h})$ ). This behaviour can be seen in Fig. 8(c). The solution traverses a small section of the $\lambda^{\prime}{ }_{1}$ dispersion curve before a jump along that curve (the semi-characteristic shock) and then the jump to the $\lambda^{\prime}{ }_{3}$ curve.

When $u_{l}$ increases further still, $\frac{d \lambda_{1}}{d F}<0$ has the same sign as $\frac{d \lambda^{\prime} 1}{d F}$. When $1.848 \lesssim u_{l} \lesssim$ 2.98 , the $\lambda_{1}$ characteristics converge, resulting in a $\lambda_{1}$ shock (structure (iv), e.g., $u_{l}=2.5$ ). We can see this behaviour in Fig. 8(d), in which there is an immediate jump along the $\lambda^{\prime}{ }_{1}$ curve, followed by the jump to the $\lambda^{\prime}{ }_{3}$ curve.

When $u_{l} \gtrsim 2.98, F_{l}>1.613$ and $h_{*}>1$. Across the $\lambda_{1}$ wave, $h$ increases and $u$ decreases, and $F$ therefore decreases. However, across the $\lambda_{1}$ wave, $F>1.613$. As $F$ decreases, $\lambda^{\prime}{ }_{1}$ increases (Fig. 5(e)), and therefore $\lambda_{1}$ also increases. Thus the $\lambda_{1}$ wave is a rarefaction (structure (v), e.g., $u_{l}=3.5$ ). The decreasing $F$ results in the "reversal" of the path of the wave in $\lambda^{\prime}-F$ space: see Fig. $8(\mathrm{e})$.

Finally, the results show that the $\lambda_{3}$ wave is always a rarefaction fan, although it is not immediately clear that $\lambda_{3}$ increases from the relation $\lambda_{3}=\lambda^{\prime}{ }_{3} \sqrt{h}$. However, in the limit $F \rightarrow \infty$, Eq. (12) can be factorised such that $\lambda^{\prime}{ }_{3} \sim F, \Rightarrow \lambda_{3} \sim u$. The $\lambda_{3}$ wave is a $\lambda_{-}$ hydrodynamic wave, and it is a fan when $h$ decreases from left to right.

\section{B Interpretation of wave structures in Sect. 3.2.1 by characteristics}

The Riemann solutions in Fig. 6 are mapped onto $\left(\lambda^{\prime}, F\right)$ space in Fig. 9, as the solutions are traversed from left to right.

The $\lambda_{1}$ characteristics across the $\lambda_{1}$ wave for varying $u_{l}$ are not analysed here, because they are very similar to those in the wet-dry problem in Sect. 3.1. However, we can see that there is a difference in the $\lambda_{1}$ wave type because of the difference between $\mathbf{U}_{l *}$ and $\mathbf{U}_{*}$.

Across the $\lambda_{3}$ wave, we have $\frac{d \lambda_{3}^{\prime}{ }_{3}}{d F}>0$. In structure (i)-(ii), $h$ and $F$ both decrease.

However, it is not clear whether $\lambda_{3}=\lambda^{\prime}{ }_{3} \sqrt{h}$ increases or decreases because $\lambda^{\prime}{ }_{3}<0$. The $\lambda_{3}$ wave is a $\lambda_{+}$wave in structure (i) and a combination of the $\lambda_{+}$hydrodynamic wave 
and morphodynamic wave in structure (ii). In structure (ii), the $\lambda_{+}$wave is more dominant. Therefore, it is a shock because $h$ decreases from left to right across the wave.

In structure (iii), $F$ increases across the $\lambda_{3}$ wave. The $\lambda_{3}$ wave in structure (iii) is a morphodynamic wave, and because $F$ is close to 0 where $\frac{d \lambda^{\prime}}{d F}=0, \frac{d \lambda_{3}}{d F}$ could have different signs from $\frac{d \lambda^{\prime}}{d F}$ (Sect. 2.5). The results show that $\frac{d \lambda_{3}}{d F}<0$, and the $\lambda_{3}$ wave is a shock.

The $\lambda_{3}$ wave in structure (iv)-(vi) is a $\lambda_{-}$wave or a combination of the $\lambda_{-}$hydrodynamic wave and morphodynamic wave (Fig. 9). In structure (iv)-(v), $h$ decreases and $F$ increases across the $\lambda_{3}$ wave (Fig. 6(e) and Fig. 9(g)). Across the morphodynamic part of $\lambda_{3}$ wave in structure (iv)-(v), $\frac{d \lambda_{3}}{d F}>0$ has the same sign with $\frac{d \lambda_{3}^{\prime}}{d F}$ because it is not close to $\frac{d \lambda^{\prime}}{d F}=$ 0 (i.e., $F=0$ ). Thus, the morphodynamic part is a rarefaction. The $\lambda_{-}$wave is also a rarefaction because $h$ decreases. Therefore, the $\lambda_{3}$ wave is a rarefaction in structure (iv)(v). However, in structure (vi), $h$ increases across the $\lambda_{3}$ wave (Fig. 6 (e)), and the $\lambda_{3}$ wave is a shock.

In structure (i)-(ii), the $\lambda_{2}$ wave is a combination of $\lambda_{+}$hydrodynamic wave and morphodynamic wave. As water depth increases from left to right, the $\lambda_{+}$wave is a rarefaction. However, as $F$ is close to -1.613 where $\frac{d \lambda_{2}^{\prime}}{d F}=0$, the morphodynamic part $\frac{d \lambda_{m}}{d F}$ changes its sign. The morphodynamic part is a combination of a rarefaction and a semi-characteristic in structure (i) and a rarefaction in structure (ii). Therefore, the $\lambda_{2}$ wave is a combination of a rarefaction and a shock in structure (i), and a rarefaction in structure (ii).

We know that $\frac{d \lambda_{2}^{\prime}}{d F}>0$ when $F \gtrsim-1.613$, and $\frac{d \lambda^{\prime} 2}{d F}<0$ when $F \lesssim-1.613$. In structure (iii)-(vi), $h$ and $F$ decrease across the $\lambda_{2}$ wave with $F>-1.613$. Therefore, $\lambda^{\prime}{ }_{2}$ and $\lambda_{2}=\lambda^{\prime}{ }_{2} \sqrt{h}$ both decrease, and the $\lambda_{2}$ wave is a shock. From the physical perspective, the $\lambda_{2}$ wave in structure (iii)-(vi) is a $\lambda_{+}$wave, and it is a shock when $h$ decreases.

\section{Interpretation of wave structures in Sect. 3.2.2 by characteristics}

The Riemann solutions in Fig. 7 are mapped onto $\left(\lambda^{\prime}, F\right)$ space in Fig. 10, as the solutions are traversed from left to right. We can see the black solid line starts from $F_{l}$ along the $\lambda^{\prime}{ }_{1}$ curve, and ends at $F_{r}$ on the $\lambda_{2}$ curve, with a jump between $\lambda^{\prime}{ }_{1}$ and $\lambda^{\prime}{ }_{3}\left(\lambda^{\prime}{ }_{3}\right.$ and $\left.\lambda_{2}^{\prime}{ }_{2}\right)$ curves through the left (right) star region.

In structure (i)-(ii), across the $\lambda_{1,3}$ wave, $h$ increases, $u$ decreases and $F$ decreases (Fig. 10(f)). Therefore, $\lambda^{\prime}{ }_{1}$ decreases. We can deduce that $\lambda_{1}$ decreases from $\lambda_{1}=\lambda^{\prime}{ }_{1} \sqrt{h}$ because $\lambda^{\prime}{ }_{1}<0$, and the $\lambda_{1}$ wave is a shock. The $\lambda_{1}$ wave is a $\lambda_{-}$hydrodynamic shock, and it is a shock when water depth increases from left to right.

In structure (i)-(ii), $\lambda^{\prime}{ }_{3}<0$ decreases as $F$ decreases across the $\lambda_{3}$ wave (Fig. 10(a)-(c)). In structure (i), the $\lambda_{3}$ wave is a combination of $\lambda_{+}$hydrodynamic wave and morphodynamic wave. Because $h$ decreases, the hydrodynamic wave in structure (i) is a shock. The morphodynamic part is overtaken by the hydrodynamic shock, and the $\lambda_{3}$ wave in structure (i) is a shock. While in structure (ii), it is a morphodynamic wave, and $\frac{d \lambda_{3}}{d F}$ has the same sign as that of $\frac{d \lambda^{\prime}}{d F}$ resulting in a $\lambda_{3}$ shock.

The $\lambda_{2}$ wave in structure (i) is a morphodynamic wave. Therefore $\frac{d \lambda_{2}}{d F}<0$ because $\frac{d \lambda^{\prime} 2}{d F}<0$ and $F$ is far from -1.613 where $\frac{d \lambda^{\prime} 2}{d F}=0$. When $F$ decreases, $\lambda_{2}$ increases, and hence it is a rarefaction. In structure (ii), the $\lambda_{2}$ wave is a combination of the $\lambda_{+}$wave and morphodynamic wave with $\left|F_{* r}\right| \ll\left|F_{r}\right|$ and $\lambda^{\prime}{ }_{2 * r}>\lambda_{2 r}^{\prime}$ (Fig. 10(c)). Water depth decreases from left to right across the $\lambda_{+}$wave, so it is a shock.

In structure (iii)-(iv) (e.g., $\left.u_{r}=0,1.5\right), h$ decreases and $u$ and $F$ increase across the $\lambda_{1}$ wave (Fig. 10(d)-(e)). Similar to the wet-dry dam-break solution, the $\lambda_{1}$ and $\lambda_{3}$ waves are both rarefactions.

In structure (iii), across the $\lambda_{2}$ wave, if it is a rarefaction, $h$ decreases, $u$ decreases and $F$ decreases (Fig. 7 (e)-(f) and Fig. 10(f)). Therefore $\lambda^{\prime}{ }_{2}$ decreases because $F \gtrsim-1.613$ (Fig. 10), and therefore $\lambda_{2}$ is a shock. However, in structure (iv), across the $\lambda_{2}$ wave, $h$ increases, $u$ increases and $F$ decreases (Fig. 10(f)), and therefore $\lambda^{\prime}{ }_{2}$ decreases. It is not clear whether $\lambda_{2}$ should increase or decrease across the $\lambda_{2}$ wave. From the physical perspective, 
the $\lambda_{2}$ wave is a $\lambda_{+}$hydrodynamic wave, and it is a rarefaction if water depth increases from left to right. The results show that $\lambda_{2}$ increases across the $\lambda_{2}$ wave, and it is a rarefaction.

\section{Numerical investigation of wave structures for $u_{r}=-1.933$ and $u_{r}=-1.933$ in Sect. 3.2.2}

We solve the Riemann problem from right to left. The difference $(\delta B)$ between the calculated $\left(B_{l c}\right)$ and the already known $\left(B_{l}\right)$ bed levels in the left constant region, i.e., $\delta B=B_{l c}-B_{l}$, is plotted against $h_{r *}$ for various $u_{r}$ values in Fig. 11. As mentioned, the two bed levels $\left(B_{l c}\right.$ and $\left.B_{l}\right)$ must agree within the desired accuracy, and $\delta B=0$ corresponds to the possible physical solution for $h_{r *}$. From the plot, we can see that when $u_{r} \gtrsim-1.933$, there are three roots, and the largest root is the physical solution to $h_{r *}$. As $u_{r}$ decreases, $h_{r *}$ also decreases (see Fig. 11), and the two roots coalesce around 0.65 when $u_{r} \approx-1.933$. When $u_{r} \lesssim-1.933$, there is only one root, which is $<0.1$. However, the $\lambda_{2}$ shock corresponding to this root, is unphysical because of divergence of $\lambda_{2}$ characteristics. Therefore, the $\lambda_{2}$ wave becomes a rarefaction when $u_{r} \lesssim-1.934$.

\section{References}

1. Bernetti, R., Titarev, V.A., Toro, E.F.: Exact solution of the riemann problem for the shallow water equations with discontinuous bottom geometry. J. Comput. Phys. 227, 3212-3243 (2008)

2. Grass, A.: Sediment transport by waves and currents. Technical Report FL29, University College London, London Centre for Marine Technology (1981)

3. Jeffrey, A.: Quasilinear hyperbolic systems and waves. Pitman (1976)

4. Kelly, D.M., Dodd, N.: Floating grid characteristics method for unsteady flow over a mobile bed. Computers and Fluids 38, 899-909 (2009)

5. Kelly, D.M., Dodd, N.: Beach face evolution in the swash zone. J. Fluid Mech. 661, 316-340 (2010)

6. Larson, M., Sunamura, T.: Laboratory experiment on flow characteristics at a beach step. J. Sedimentary Petrology 63(3), 495-500 (1993)

7. Lax, P.D.: Hyperbolic systems of conservation laws and the mathematical theory of shock waves. CBMS Regional Conf. Ser. Appl. Math. S.I.A.M. (1973)

8. Needham, D.J., Hey, R.D.: On nonlinear simple waves in alluvial river flows: a theory for sediment bores. Phil. Trans. Roy. Soc. Lond. A 334, 25-53 (1991)

9. Peregrine, D.H.: Equations for water waves and the approximations behind them. In: R. Meyer (ed.) Waves on Beaches and Resulting Sediment Transport, pp. 95-121. Academic Press (1972)

10. Peregrine, D.H., Williams, S.M.: Swash overtopping a truncated beach. J. Fluid Mech. 440, 391-399 (2001)

11. Pritchard, D., Hogg, A.J.: On sediment transport under dam-break flow. J. Fluid Mech. 473, 265-274 (2002)

12. Pritchard, D., Hogg, A.J.: On the transport of suspended sediment by a swash event on a plane beach. Coastal Eng. 52, 1-23 (2005)

13. Ritter, A.: Die fortpflanzung der wasserwellen. Vereine Deutcher Ingenieure Zeitschrift 36, 947-954 (1892)

14. S., K., Ozmen-Cagatay, H.: Investigation of dam-break induced shock waves impact on a vertical wall. Journal of Hydrology 525, 1-12 (2015)

15. Stoker, J.: Water Waves. Interscience, New York, N.Y. (1957)

16. Toro, E.F.: Shock-capturing methods for free-surface shallow flows. Wiley, New York, NY. (2001)

17. Toro, E.F.: Riemann solvers and numerical methods for fluid dynamics, 3rd edn. Springer, Berlin (2009)

18. Valiani, A., Caleffi, V.: Momentum balance in the shallow water equations on bottom discontinuities. Advances in Water Resources 100, 1-13 (2017) 
26 19. Whitham, G.B.: Linear and Nonlinear Waves. Wiley-Interscience, New York (1974)

20. Zhu, F., Dodd, N.: Net beach change in the swash: A numerical investigation. Advances in Water Resources 53, 12-22 (2013)

21. Zhu, F., Dodd, N.: The morphodynamics of a swash event on an erodible beach. J. Fluid Mech. 762, 110-140 (2015)

22. Zhu, F., Dodd, N.: Riemann solution for a class of morphodynamic shallow water dambreak problems. J. Fluid Mech. 835, 1022-1047 (2018)

23. Zhu, F., Dodd, N., Briganti, R.: Impact of a uniform bore on an erodible beach. Coastal Eng. 60, 326-333 (2012) 

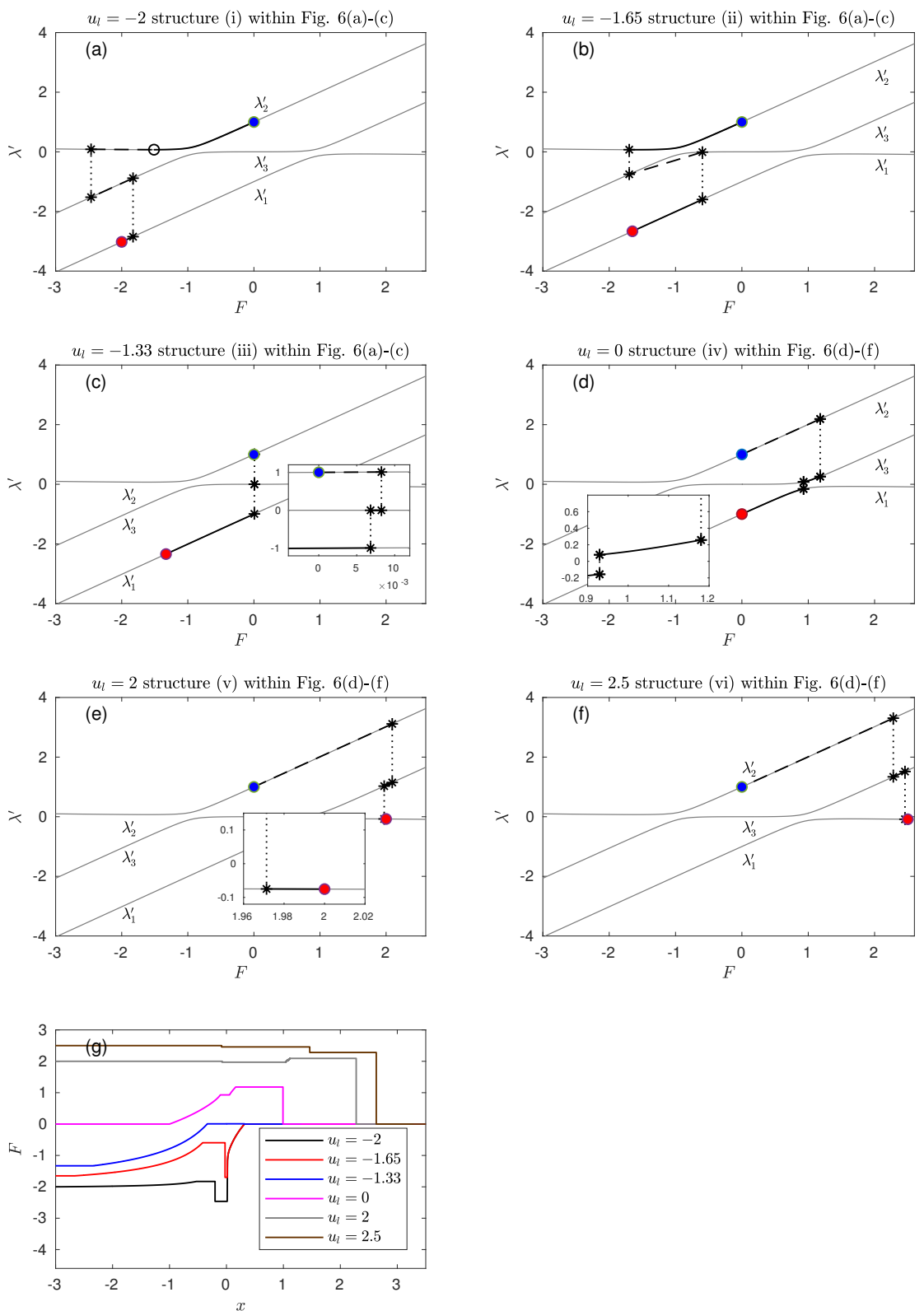

Fig. 9 (a)-(f): Illustrations of the Riemann solutions depicted in Fig. 6 in $\left(\lambda^{\prime}, F\right)$ space as the solutions are traversed from left (indicated by the red filled circle) to right (indicated by the blue filled circle). Dashed lines indicate jumps at shocks or semi-characteristic shocks. 0 separates the rarefaction and semi-characteristic shock of the same wave. Dotted lines with black $*$ represent jumps from different characteristic families of waves in star regions. $(\mathrm{g})$ : Illustration of how $F$ varies across these solutions. 

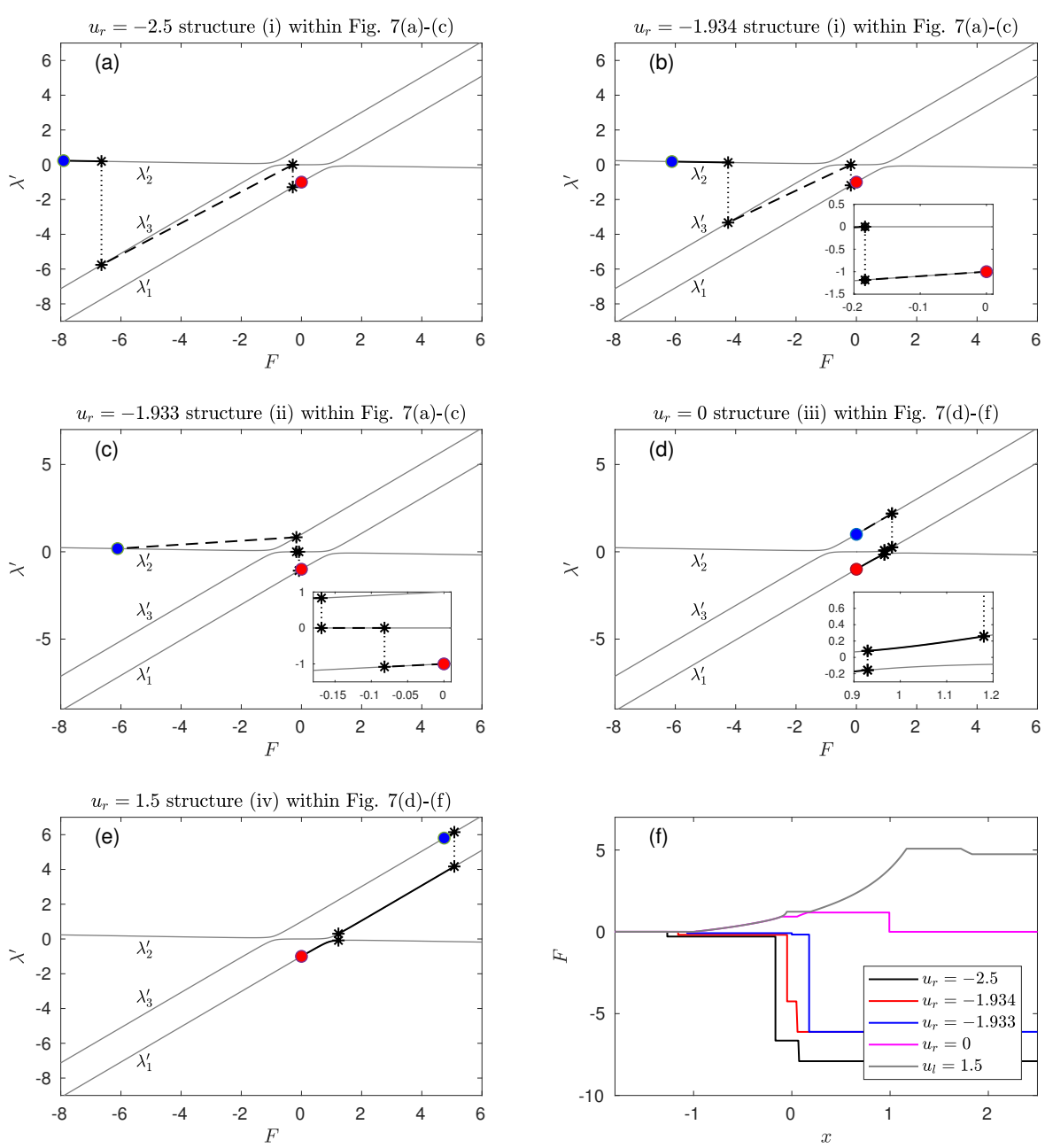

Fig. 10 (a)-(e): Illustrations of the Riemann solutions depicted in Fig. 7 in $\left(\lambda^{\prime}, F\right)$ space as the solutions are traversed from left (indicated by the red filled circle) to right (indicated by the blue filled circle). Dashed lines indicate jumps at shocks or semi-characteristic shocks. $\circ$ separates the rarefaction and semi-characteristic shock of the same wave. Dotted lines with black * represent jumps from different characteristic families of waves in star regions. (f): Illustration of how $F$ varies across these solutions. 


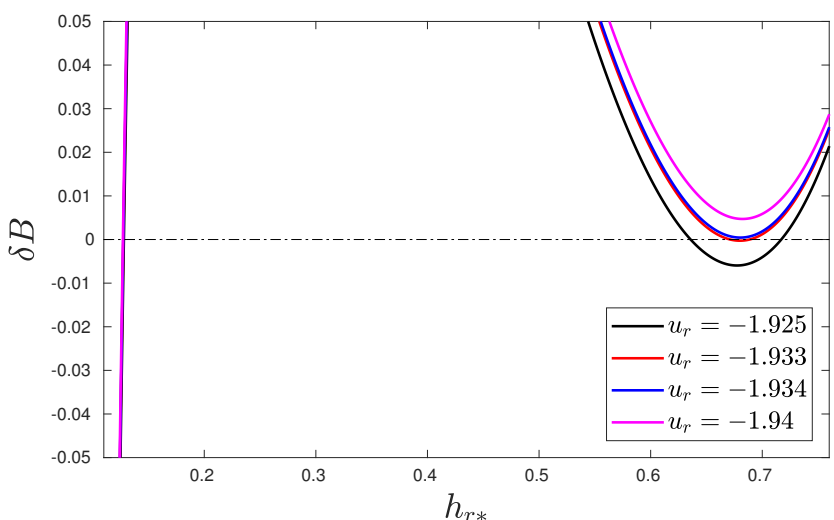

Fig. 11 Difference $(\delta B)$ between the calculated and the already known bed levels in the left constant region as a function of water depth in the right star region $\left(h_{r *}\right)$ for various $u_{r}$ values. 